\title{
A SVT-ESPRIT Estimation Algorithm in Sparse Array
}

\author{
Wenhao Zeng ${ }^{1, ~ a}$, Junjie $\mathrm{He}^{2,}$, , Hongtao $\mathrm{Li}^{1,{ }^{,},}{ }^{*}$, Xiaohua Zhu ${ }^{1, \mathrm{~d}}$ \\ ${ }_{1}^{1}$ Nanjing University of Science and Technology, Nanjing 210094, China;
}

2 The 28th Research Institute of China Electronics Technology Group Corporation, Nanjing 210094, China.

atrikona54@163.com, bhjj19900202@sina.com, cliht@njust.edu.cn, dzxh@mail.njust.edu.cn

\begin{abstract}
With regard to two dimensional Direction-of-Arrival (2-D DOA) estimation in sparse array, this paper presents a singular value threshold based estimation of signal parameters via rotational invariance technique (SVT-ESPRIT) algorithm. Firstly, a signal model of DOA estimation based on matrix completion is established which turns out to meet Null Space Property (NSP). Secondly, a full signal array is recovered from a sparse signal array using fixed-point iteration algorithm. Finally, 2-D DOA estimation can be achieved from the recovered signal. The proposed algorithm can reduce the number of array elements and achieve high accuracy of 2-D DOA estimation in sparse array. Computer simulations demonstrate the effectiveness and robustness of the proposed algorithm.
\end{abstract}

Keywords: Array signal processing; Sparse planar array; DOA Estimation; Matrix Completion.

\section{Introduction}

DOA estimation is an integral part of array signal processing, and has been applied in wide-range fields, including digital communication, signal processing, target recognition and so on [1-3]. Comparatively, 2-D DOA estimation $[4,5]$ performs better because of the fact that it can obtain both target azimuth and pitch angle at the same time. In planar array [6], 2-D DOA estimation can obtain target angle with higher accuracy, lower average side lobes, and it is not sensible to noise. However, in this case, considerable hardware equipment is required, such as antennas, sampling apparatus, etc, which increases cost significantly. By contrast, in sparse array, the number of array units, antennas, sampling devices can be effectively declined and design cost can be largely cut down. Nevertheless, for this case, the problem of angle ambiguity occurs and average side lobes of frequency spectrum may rise substantially.

Matrix completion [7-9] is an extension of compressive sensing (CS) [10,11]. If a matrix is a low-rank matrix, matrix completion can reconstruct a full matrix from a subset of array elements. This theory has been widely adopted in image processing [15], pattern recognition [16], etc. Matrix completion technique converts the problem of least square to the problem of nuclear-norm optimization which lowers the complexity of matrix reconstruction. In recent years, this technique develops rapidly and has achieved impressive breakthroughs in many fields, involving in Fast Algorithm [12, 13], Exact Recovery [14] and so on.

This paper presents a singular value threshold based estimation of signal parameters via rotational invariance technique (SVT-ESPRIT) algorithm. According to low-rank property of two dimensional receive signal array, the proposed algorithm firstly formulates a signal model of DOA estimation based on matrix completion and this model is proved to meet null space property (NSP). Thus, the robustness of DOA estimation via matrix completion is ensured. Secondly, a full array signal is recovered from a sparse array signal by fixed-point iterative method. Finally, 2-D DOA estimation can be acquired from the recovered signal by estimation of signal parameters via rotational invariance technique (ESPRIT) algorithm. By contrast to conventional algorithms, the proposed algorithm combines matrix completion with 2-D DOA estimation which can estimate target angle precisely and meantime enhance the availability of array elements. 


\section{Signal Model in Sparse Array}

Suppose uniform rectangular array (URA) [3] is shown as Fig.1. The number of elements in x-direction and y-direction are $M_{x}$ and $M_{y}$ respectively. The distances between corresponding elements are $d_{x}$ and $d_{y}$. The pitch and azimuth angle of target are $\phi$ and $\theta$, respectively.

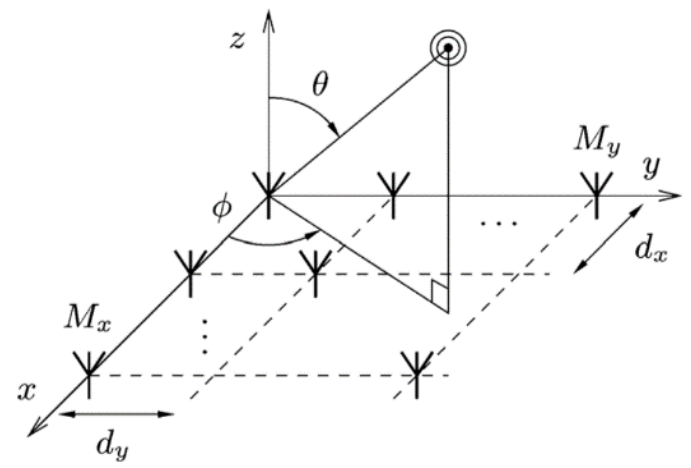

Fig. 1 The Uniform Rectangular Array

In multiple-targets scene, vector of receive signal model is expressed as $X(t)=A_{x} S(t) A_{y}^{T}+N_{R}(t), t=1, \ldots, N$

Where $N$ is the number of snapshot, $\eta$ is the number of target, $s_{i}(t)$ is waveform of the i-th target and $n(t)$ is measurement noise of dimension $M_{x} M_{y} \times 1, a\left(\varphi_{x i}, \varphi_{y i}\right)$ is dimensional steering vector $M_{x} M_{y} \times 1$ of the $i$-th target and corresponds to target angel in two-dimensional space domain, which can be shown as

$a\left(\varphi_{x i}, \varphi_{y i}\right)=a_{y}\left(\varphi_{y i}\right) \otimes a_{x}\left(\varphi_{x i}\right)$

Where $\otimes$ represents Kronecker products, $a_{x}\left(\varphi_{x i}\right)$ and $a_{y}\left(\varphi_{y i}\right)$ can be respectively defined as

$\left\{\begin{array}{l}{\left[a_{x}\left(\varphi_{x i}\right)\right]_{m_{x}}=e^{j \varphi_{i x}\left(m_{x}-1\right)}, m_{x}=1,2, \cdots, M_{x},} \\ {\left[a_{y}\left(\varphi_{y i}\right)\right]_{m_{y}}=e^{j \varphi_{y i}\left(m_{y}-1\right)}, m_{y}=1,2, \cdots, M_{y} .}\end{array}\right.$

Where $\varphi_{x i}$ and $\varphi_{y i}$ is determined by azimuth $\phi_{i}$ and pitch angle $\theta_{i}$ respectively as follows:

$\left\{\begin{array}{l}\varphi_{x i}=k d_{x} \sin \left(\theta_{i}\right) \cos \left(\phi_{i}\right), \\ \varphi_{y i}=k d_{y} \sin \left(\theta_{i}\right) \sin \left(\phi_{i}\right)\end{array}\right.$

Where $k=\frac{2 \pi}{\lambda}$ and $\lambda$ refers to wavelength. Convert signal model to a matrix, we have

$$
X(t)=\sum_{i=1}^{\eta} A\left(\varphi_{x i}, \varphi_{y i}\right) s_{i}(t)+N_{R}(t), t=1, \ldots, N
$$

Where $\eta$ is the number of targets, $s_{i}(t)$ is target waveform of the $i$-th target, $N_{R}(t)$ is dimensional noise matrix $M_{x} \times M_{y}$ of receive signal. $A\left(\varphi_{x i}, \varphi_{y i}\right)$ is dimensional steering matrix $M_{x} \times M_{y}$ of the $i$-th target which can be described as

$$
A\left(\varphi_{x i}, \varphi_{y i}\right)=a_{x}\left(\varphi_{x i}\right) \bullet a_{y}\left(\varphi_{y i}\right)^{T}
$$

Vectorize matrix of signal model and the vector we get is same as vector of signal model, namely $x(t)=\operatorname{vec}[X(t)]$

Matrix of signal model can be presented as

$$
X(t)=A_{x} S(t) A_{y}{ }^{T}+N_{R}(t), t=1, \ldots, N
$$

Where $\mathrm{A}_{\mathrm{x}}=\left[\mathrm{a}_{\mathrm{x}}\left(\varphi_{\mathrm{x} 1}\right), \mathrm{a}_{\mathrm{x}}\left(\varphi_{\mathrm{x} 2}\right) \cdots \mathrm{a}_{\mathrm{x}}\left(\varphi_{\mathrm{x} \eta}\right)\right] \quad$ is $M_{x} \times \eta$ dimensional steering vector, $A_{y}=\left[a_{y}\left(\varphi_{y 1}\right), a_{y}\left(\varphi_{y 2}\right) \cdots a_{y}\left(\varphi_{y \eta}\right)\right]$ is $M_{y} \times \eta$ dimensional steering vector, $S(t)=\operatorname{diag}\left(s_{i}(t)\right)$ is a diagonal matrix of $\eta \times \eta$ dimension, the first $\eta$ elements of $s_{i}(t)$ are non-zero corresponding to the number of $\eta$ targets. When the power of noise matrix $N_{R}(t)$ is much smaller than the signal power, the rank of receive signal matrix is $\operatorname{rank}(X(t))=\operatorname{rank}(S(t)) \approx \eta$, in other words, matrix $X(t)$ is low-rank. Matrix in 
Eq. (8) meets strong irrelevance property and can recover a full matrix with high accuracy via matrix completion [19].

When the number of known elements in matrix is $m \geq k r\left(n_{1}+n_{2}-r\right)$ [9], target matrix can be precisely recovered by matrix completion, where $n_{1}$ and $n_{2}$ are the number of rows and columns, respectively, $r$ is rank of target matrix and $k$ is constant. Thus, the proposed sparse array model in this paper is as follows. Select elements randomly from URA with a total number of $m$ elements to build a new sparse array. Consider receive signal model in sparse array is $X_{s}(t)$, then it is related to receive signal matrix $X(t)$ of URA depicted in Fig. 1 by

$$
\left\{\begin{array}{c}
X_{s}(t)_{i j}=X(t)_{i j},(i, j) \in \Omega \\
X_{s}(t)_{i j}=0 \quad,(i, j) \notin \Omega
\end{array}\right.
$$

Where $\Omega$ is block of array unit positions in sparse array.

\section{The Proposed Algorithm}

\subsection{Signal Model of 2-D DOA Estimation Based on Matrix Completion}

When the number of sampled elements is $m<r\left(n_{1}+n_{2}-r\right)$, it's unlikely to recover original matrix from sampled matrix using matrix completion [9]. Hence, this paper adopts the following approaches to construct a sampled matrix. Let the number of sampled elements be $m \geq 3 * r\left(n_{1}+n_{2}-r\right)$. We sample elements from the full array uniformly and randomly to formulate a sparse array where the number of elements is $m$. Consider receive signal matrix of the full array as $X(t)$ and the proposed receive signal matrix in sparse array as $X_{s}(t)$, we have

$$
P_{\Omega}(X(t))=P_{\Omega}\left(X_{s}(t)\right)
$$

Where $P_{\Omega}(X(t))$ denotes real position of antennas in sparse array.

Since $X(t)$ is low-rank matrix, we can easily note that $X_{R}(t)$ can be recovered from receive signal $X_{s}(t)$ of the sparse array [9]. In this case, the number of antennas and sampling devices are declined and the complexity of system is lowered. The corresponding matrix completion model can be summarized as

$$
\left\{\begin{array}{cc}
\min & \left\|X_{R}(t)\right\|_{*}, \\
\text { s.t. } & P_{\Omega}\left(X_{R}(t)\right)=P_{\Omega}\left(X_{s}(t)\right) .
\end{array}\right.
$$

It can be proved that if a sampling model meet NPS [10], rank minimization of this model equals to its nuclear-norm minimization. Regard null space of sampling operator $P_{\Omega}$ as

$$
\operatorname{Null}\left(P_{\Omega}\right)=\left\{M \in R^{n_{1} \times n_{2}}: P_{\Omega}(M)=0\right\},
$$

Matrix in null space $\operatorname{Null}\left(P_{\Omega}\right)$ can hardly be recovered. Analyze signal model (3) and it can be observed that

$$
\left[a_{x}\left(\varphi_{x i}\right)\right]_{m_{x}}=e^{j \varphi_{x i}\left(m_{x}-1\right)} \neq 0
$$

Arbitrary elements of both matrix $A_{x}=\left[a_{x}\left(\varphi_{x 1}\right), a_{x}\left(\varphi_{x 2}\right), \cdots, a_{x}\left(\varphi_{x \eta}\right)\right]$ and matrix $A_{y}$ in (8) are nonzero. The first $i$ elements of diagonal element $S(t)$ are nonzero. On the basis of matrix multiplication, we can know that arbitrary elements of matrix $X_{R}$ are nonzero. Thus, null space of sampling operator is a null block and whichever sampling operator is adopted, matrix $X_{R}$ won't appear in null space of sampling operator, namely $P_{\Omega}\left(x_{\mathrm{R}}\right) \notin \operatorname{Null}\left(P_{\Omega}\right)$ and matrix $X_{R}$ meets NSP.

\subsection{SVT-ESPRIT Algorithm}

2-D DOA estimation in planar array can locate target angle precisely with lower average side lobes and not easily interfered by noise and clutter. Nevertheless, massive hardware equipment, like antennas and sampling devices, is necessary in this case which results in significantly increased cost. In comparison, 2-D DOA estimation in sparse array performs effectively in reducing the number of array units, antennas as well as sampling devices and cutting down design cost. However, on this occasion, accuracy of target estimation declines and spectrum average side lobes rise substantially.

In view of the foregoing problem, this paper proposes a SVT-ESPRIT algorithm. Firstly, estimation matrix $X_{R}(t)$ of full matrix is recovered from receive signal matrix $X_{s}(t)$ of sparse array 
using matrix completion. Secondly, resorting to spectrum estimation algorithm, 2-D DOA estimation of target is obtained. Neglect noise interference and then vector of receive signal is

$$
X_{\mathrm{R}}(t)=A_{x} S(t) A_{y}^{T}
$$

Let $A_{x 1}=\left[a_{x 1}\left(\phi_{1}, \theta_{1}\right), a_{x 1}\left(\phi_{2}, \theta_{2}\right), \cdots, a_{x 1}\left(\phi_{k}, \theta_{k}\right)\right]$ and $A_{x 2}=\left[a_{x 2}\left(\phi_{1}, \theta_{1}\right), a_{x 2}\left(\phi_{2}, \theta_{2}\right), \cdots, a_{x 2}\left(\phi_{k}, \theta_{k}\right)\right]$, where

$$
\left\{\begin{array}{c}
{\left[a_{x 1}\left(\phi_{i}, \theta_{i}\right)\right]_{m_{x}}=e^{j \frac{2 \pi}{\lambda} d_{x} \sin \left(\theta_{i}\right) \cos \left(\phi_{i}\right)\left(m_{x}-1\right)}, m_{x}=1,2, \cdots, M_{x}-1} \\
{\left[a_{x 2}\left(\phi_{i}, \theta_{i}\right)\right]_{m_{x}}=e^{j \frac{2 \pi}{\lambda} d_{x} \sin \left(\theta_{i}\right) \cos \left(\phi_{i}\right)\left(m_{x}-1\right)}, m_{x}=2,3, \cdots, M_{x}}
\end{array}\right.
$$

There is one less twiddle factor in $A_{x 1}$ than $A_{x 2}$ and $A_{x 2}=A_{x 1} \Phi_{x}$ where

$$
\Phi_{x}=\operatorname{diag}\left\{e^{j \frac{2 \pi}{\lambda} d_{x} \sin \left(\theta_{1}\right) \cos \left(\phi_{1}\right)}, e^{j \frac{2 \pi}{\lambda} d_{x} \sin \left(\theta_{2}\right) \cos \left(\phi_{2}\right)}, \cdots e^{j \frac{2 \pi}{\lambda} d_{x} \sin \left(\theta_{k}\right) \cos \left(\phi_{k}\right)}\right\}
$$

Suppose subspace of signal is $E_{s}$ and

$$
\left\{\begin{array}{c}
E_{1}=E_{s}\left(1:\left(M_{x}-1\right) M_{y},:\right) \\
E_{2}=E_{s}\left(M_{y}+1: M_{x} M_{y},:\right)
\end{array}\right.
$$

Where $E_{1}$ is related to $E_{2}$ by

$$
\left[\begin{array}{c}
E_{1} \\
E_{2}
\end{array}\right]=\left[\begin{array}{c}
A_{x 1} S(t) A_{y}{ }^{T} \\
A_{x 1} \Phi_{x} S(t) A_{y}{ }^{T}
\end{array}\right]
$$

Thus,

$$
E_{2}=E_{1} T_{1}^{-1} \Phi_{x} T_{1}=E_{1} \psi_{x}
$$

Where $\psi_{x}=T_{1}^{-1} \Phi_{x} T_{1}$ and $T$ is a full-rank matrix. Based on least square method, it can be concluded that

$\hat{\psi}_{x}=E_{S 1}{ }^{+} E_{S 2}$

Eigen-decompose $\hat{\psi}_{x}$ and we have $\Phi_{x}$. From (16), it can be deduced that

$r_{x}=\left[\sin \theta_{1} \cos \phi_{1}, \sin \theta_{2} \cos \phi_{2}, \cdots, \sin \theta_{k} \cos \phi_{k}\right]$

Transpose matrix $X_{\mathrm{R}}(t)$ and likewise we have

$r_{y}=\left[\sin \theta_{1} \sin \phi_{1}, \sin \theta_{2} \sin \phi_{2}, \cdots, \sin \theta_{k} \sin \phi_{k}\right]$

After angle matching, DOA estimation of target is

$$
\left\{\begin{array}{c}
{\left[\theta_{1}, \theta_{2}, \cdots, \theta_{k}\right]=\arcsin \sqrt{r_{x}^{2}+r_{y}^{2}}} \\
{\left[\phi_{1}, \phi_{2}, \cdots, \phi_{k}\right]=\arctan \left(r_{\mathrm{y}} / r_{\mathrm{x}}\right)}
\end{array}\right.
$$

\section{Simulations}

In this section, some simulations are conducted to demonstrate the robustness of 2-D DOA estimation in sparse array and the effectiveness of the proposed algorithm. In the simulations, full array is a URA of $64 \times 64$ elements with the total number of array elements $m=4096$ and elements distance in $\mathrm{x}$-direction and $\mathrm{y}$-direction $d_{x}=d_{y}=\lambda / 2$. However, sparse array is constructed from randomly sampled elements of full array where the total number of sampled units is 1200 . There are two targets in space domain and their corresponding 2-D DOA are $\left(30^{\circ}, 15^{\circ}\right)$ and $\left(10^{\circ}, 35^{\circ}\right)$, respectively.

Experiment 1: 2-D DOA estimation in sparse array based on matrix completion is analyzed. Let the number of snapshots be 100, SNR is $20 \mathrm{~dB}$ and 500 Monte Carlo simulations are implemented. Fig. 2 shows the simulation result of 2-D DOA estimation using SVT-ESPRT algorithm, the proposed algorithm adopts sparse matrix where there are only 1200 array elements, in other words, $70 \%$ units removed from full array. Compared with conventional DOA estimation algorithm, the presented algorithm directly obtains receive signal from sparse array and acquires target angle by matrix completion and spectrum estimation technique, for the benefit of higher-resolution angle estimation and enhanced element availability. 


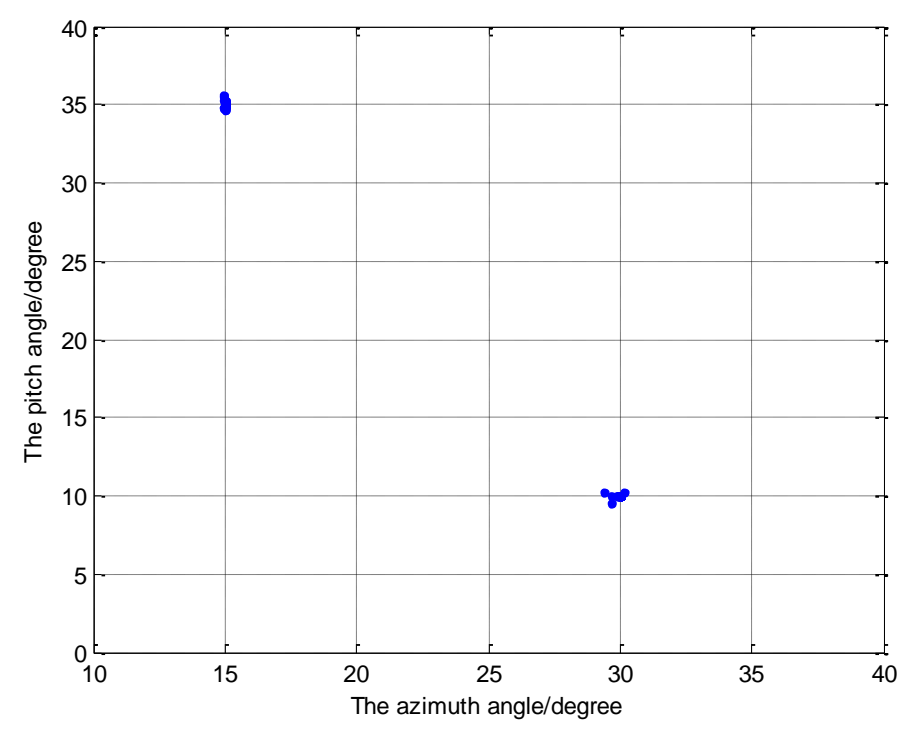

Fig. 2 2-D DOA estimation by SVT-ESPRIT Algorithm

Experiment 2: SVP-ESPRIT algorithm with different SNR and array elements is discussed. In Fig. 3 , it depicts RMSE with different SNR by SVP-ESPRIT algorithm, when the number of array elements is 900, 1200 and 1500, respectively. Let the number of snapshots is 100 and 500 Monte Carlo simulations is conducted. It is convenient to note that estimation accuracy of SVP-ESPRIT algorithm is proportional to the number of array elements. This result is due to that the recovery accuracy of matrix completion grows with the increasing number of array elements, and furthermore the spectrum estimation of higher recovery accuracy leads to smaller estimation error.

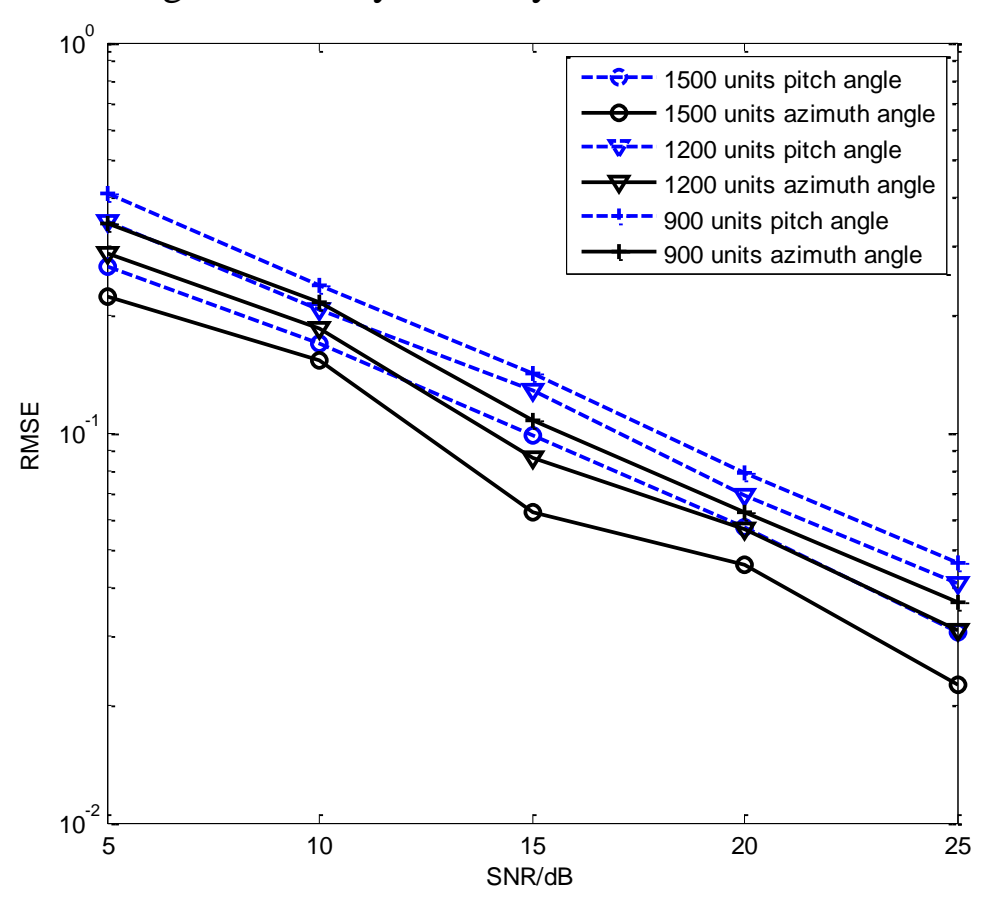

Fig. 3 RMSE against different SNR and array elements

\section{Conclusion}

This paper presents a SVT-ESPRIT algorithm. A signal model of DOA estimation based on matrix completion is established. This model proves to meet the NSP and can obtain 2-D DOA estimation effectively in sparse array. By contrast to conventional 2-D DOA estimation algorithms, the proposed algorithm directly achieves receive signal from sparse array. Besides, it acquires target angle by matrix completion and spectrum estimation technique which has high-resolution angle estimation and improved elements availability. 


\section{Acknowledgments}

This work was supported by the National Natural Science Foundation of China (Grant No.61401204), Postdoctoral Science Foundation of Jiangsu Province (Grant No. 1501104C), Technology Research and Development Program of Jiangsu Province (Grant No. BY2015004-03), and the Fundamental Research Funds for the Central Universities (Grant No. 30916011319).

\section{References}

[1] Lee J, Song I, Kwon H, et al. Low-complexity estimation of 2D DOA for coherently distributed sources. Signal processing, Vol. 83 (2003) No. 8, p. 1789-1802.

[2] Hori Y, Moriya H, Ichige K, et al. 2D-DOA estimation under LOS environment by using pyramid shaped array antenna. Electromagnetics (iWEM). 2013 IEEE International Workshop on. IEEE, 2013, p. 78-79.

[3] Wang L. Joint 2-D DOA and Noncircularity Phase Estimation Method. Journal of Radars, Vol. 1 (2012) No. 1, p.43-49.

[4] Liu Z, Ruan X, He J. Efficient 2-D DOA estimation for coherent sources with a sparse acoustic vector-sensor array. Multidimensional Systems \& Signal Processing, Vol. 24 (2013) No. 1, p. $105-120$.

[5] Zhu Y, Luo J Q. 2-D DOA Estimation Algorithm of Multiple Wideband Sources Based on Three Sensors. Fire Control \& Command Control, Vol. 38 (2013) No. 5, p. 105-108.

[6] Heidenreich P, Zoubir A M, Rubsamen M. Joint 2-D DOA estimation and phase calibration for uniform rectangular arrays. Signal Processing, IEEE Transactions on, Vol. 60 (2012) No. 9, p. 4683-4693.

[7] Candes E J, Eldar Y C, Strohmer T, et al. Phase retrieval via matrix completion[J]. SIAM Journal on Imaging Sciences, Vol. 6 (2013) No. 1, p. 199-225.

[8] Recht B, Xu W, Hassibi B. Necessary and sufficient conditions for success of the nuclear norm heuristic for rank minimization.Decision and Control, 47th IEEE Conference on. IEEE, 2008, p. 3065-3070.

[9] Candès E J, Recht B. Exact matrix completion via convex optimization. Foundations of Computational mathematics, Vol. 9 (2009) No. 6, p. 717-772.

[10]Compressed sensing: theory and applications. Cambridge University Press, 2012, p 5-15.

[11]Duarte M F, Baraniuk R G. Spectral compressive sensing. Applied and Computational Harmonic Analysis, Vol. 35 (2013) No. 1, p. 111-129.

[12] Ma S, Goldfarb D, Chen L. Fixed point and Bregman iterative methods for matrix rank minimization. Mathematical Programming, (2011) No. 1, p. 321-353.

[13] Hu Y, Zhang D, Ye J, et al. Fast and accurate matrix completion via truncated nuclear norm regularization. Pattern Analysis and Machine Intelligence, IEEE Transactions on, Vol. 35 (2013) No. 9, p. 2117-2130.

[14]Recht B. A simpler approach to matrix completion. The Journal of Machine Learning Research, Vol.12 (2011) No. 4, p. 3413-3430.

[15]Chen C, He B, Yuan X. Matrix completion via an alternating direction method. IMA Journal of Numerical Analysis, Vol. 32 (2012) No. 1, p. 227-245.

[16]Connor Schenck, Jivko Sinapov, David Johnston, et al. Which Object Fits Best? Solving Matrix Completion Tasks with a Humanoid Robot. IEEE Transactions on Autonomous Mental Development, Vol. 6, No. 3, September 2014 .p 226-240.

[17]Kalogerias, Dionysios S, Petropulu, Athina P. Matrix Completion in Colocated MIMO Radar: Recoverability, Bounds \& Theoretical Guarantees. Signal Processing, IEEE Transactions on, Vol. 62 (2014) No. 2, p. 105-120. 